\title{
(6) OPEN ACCESS \\ Polarisation-sensitive OCT is useful for evaluating retinal pigment epithelial lesions in patients with neovascular AMD
}

\author{
Christopher Schütze, ${ }^{1}$ Katharina Teleky, ${ }_{1}^{1}$ Bernhard Baumann, ${ }^{2}$ Michael Pircher, ${ }^{2}$ \\ Erich Götzinger, ${ }^{2}$ Christoph K Hitzenberger, ${ }^{2}$ Ursula Schmidt-Erfurth ${ }^{1}$
}

${ }^{1}$ Department of

Ophthalmology, Medical University of Vienna, Vienna, Austria

${ }^{2}$ Center for Medical Physics and Biomedical Engineering, Medical University of Vienna, Vienna, Austria

\section{Correspondence to} Professor Ursula SchmidtErfurth, Department of Ophthalmology, Medical University of Vienna, Waehringer Guertel 18-20, 1090 Vienna, Austria; ursula.schmidt-erfurth@ meduniwien.ac.at

Received 7 January 2015 Revised 17 April 2015 Accepted 19 June 2015 Published Online First 16 July 2015

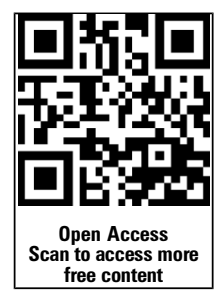

CrossMark

To cite: Schütze $C$,

Teleky K, Baumann B, et al. $\mathrm{Br} J$ Ophthalmol

2016;100:371-377.

\section{ABSTRACT}

Background/aims To examine the reproducibility of lesion dimensions of the retinal pigment epithelium (RPE) in neovascular age-related macular degeneration (AMD) with polarisation-sensitive optical coherence tomography (PS-OCT), specifically imaging the RPE. Methods Twenty-six patients (28 eyes) with neovascular AMD were included in this study, and examined by a PS-OCT prototype. Each patient was scanned five times at a 1-day visit. The PS-OCT B-scan located closest to the macular centre presenting with RPE atrophy was identified, and the longitudinal diameter of the lesion was quantified manually using AutoCAD 2008. This procedure was followed for the identical B-scan position in all five scans per eye and patient. Reproducibility of qualitative changes in PS-OCT was evaluated. Interobserver variability was assessed. Results were compared with intensity-based spectraldomain OCT (SD-OCT) imaging.

Results Mean variability of all atrophy lesion dimensions was $0.10 \mathrm{~mm}(S D \pm=0.06 \mathrm{~mm})$. Coefficient of variation ( $S D \pm /$ mean) was 0.06 on average (SD $\pm=0.03$ ). Interobserver variability assessment showed a mean difference of $0.02 \mathrm{~mm}$ across all patients regarding RPE lesion size evaluation (paired t test: $p=0.38$ ).

Spearman correlation coefficient was $r=0.98, p<0.001$. Results revealed a good overall reproducibility of $\sim 90 \%$. PS-OCT specifically detected the RPE in all eyes compared with conventional intensity-based SD-OCT that was not capable to clearly identify RPE atrophy in 25 eyes $(89.3 \%, p<0.01)$.

Conclusions PS-OCT offers good reproducibility of RPE atrophy assessment in neovascular AMD, and may be suitable for precise RPE evaluation in clinical practice. PS-OCT unambiguously identifies RPE changes in choroidal neovascularisation compared with intensity-based SD-OCT that does not identify the RPE status reliably.

\section{INTRODUCTION}

The retinal pigment epithelium (RPE) is significantly involved in the pathogenesis of neovascular age-related macular degeneration (AMD). AMDspecific alterations lead to RPE cell death and vision loss. ${ }^{1-3}$

Spectral-domain optical coherence tomography (SD-OCT) detects retinal alterations in choroidal neovascularisation (CNV). However, SD-OCT lacks RPE-specific detection because intensity-based contrast is comparatively weak, and enhanced penetration depth from RPE atrophy is not always detectable, particularly when investigating small atrophy dimensions. Polarisation-sensitive OCT
(PS-OCT) has been introduced as a relatively new imaging modality ${ }^{4-17}$ that overcomes these limitations by measuring the polarisation state of light, thereby specifically imaging the RPE based on its tissue-specific contrast. ${ }^{8-10} 12$ Melanin-containing structures like the RPE, ${ }^{9} 1016$ the iris pigment epithelium $^{14}$ or accumulations of pigment-loaded macrophages in outer retinal layers depolarise. Besides, in drusen and geographic atrophy (GA), ${ }^{13} \quad 14 \quad 17$ PS-OCT frequently shows RPE atrophy in neovascular AMD. ${ }^{11}$ Identification of disease-specific patterns in $\mathrm{CNV}$ is significant regarding the status and progression of RPE disease, potentially contributing to a better pathophysiological understanding of neovascular AMD.

Aim of this study was to examine the quantitative reproducibility of RPE atrophy dimensions in eyes with neovascular AMD using PS-OCT in addition to assess the reproducibility of qualitative changes detected by PS-OCT. Results are discussed with reference to standard imaging techniques, as well as the advantages of the specific physical properties of PS-OCT.

\section{PATIENTS AND METHODS}

Twenty-six randomly selected patients (28 eyes) with neovascular AMD previously confirmed by biomicroscopy and a standardised fluorescein angiography (FA) were included into this prospective cross-sectional trial. Patients underwent a comprehensive ophthalmological examination, including best-corrected visual acuity (BCVA), slit-lamp biomicroscopy, FA and SD-OCT (Cirrus HD-OCT; Carl Zeiss Meditec, Dublin, California, USA) prior to study participation.

The local ethics committee approved the study protocol that adhered to the ethical tenets of the Declaration of Helsinki. Each patient gave written informed consent. Following patient inclusion, specific RPE imaging by PS-OCT was performed.

Exclusion criteria were other retinal pathologies, including GA and macular dystrophies.

Four treatment-naïve patients (four eyes) and 22 patients (24 eyes) previously treated with ranibizumab (Lucentis; Novartis Pharma AG, Basel, Switzerland) were included. Earlier treatment with ranibizumab was prescribed based on routine clinical examinations. Untreated patients who were initially clinically diagnosed with neovascular AMD on the same day of PS-OCT imaging were immediately treated as needed following routine clinical BCVA assessment, biomicroscopy, FA and SD-OCT imaging. 
Figure 1 Five repeated polarisation-sensitive optical coherence tomography (PS-OCT) measurements (1-5) of a patient diagnosed with choroidal neovascularisation secondary to neovascular age-related macular degeneration that show zones of retinal pigment epithelial (RPE) atrophy. Left: intensity B-scans with RPE overlay; middle: corresponding degree of polarisation uniformity images with respective RPE atrophy lesions; right: enlarged views of repeated measurements showing the lesion extensions and the manual segmentation lines (black). Rectangles represent sectors of PS-OCT B-scans comprising RPE atrophy and measured RPE atrophy section lengths, enlarged on the right.
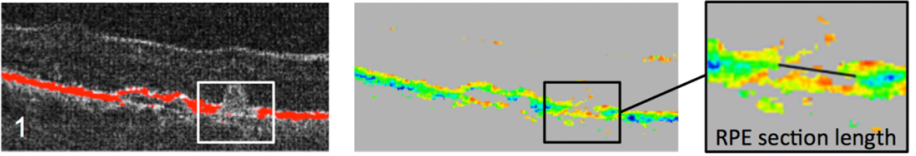

$0.31 \mathrm{~mm}$
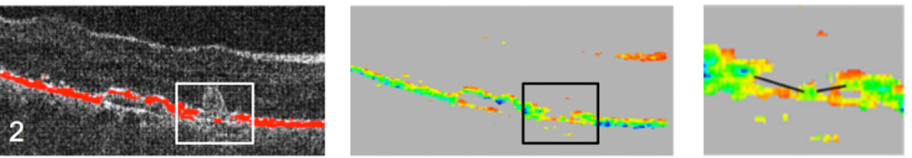

$0.33 \mathrm{~mm}$
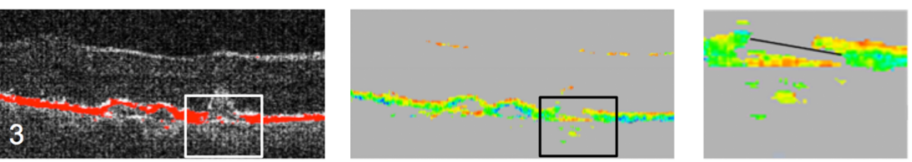

$0.36 \mathrm{~mm}$
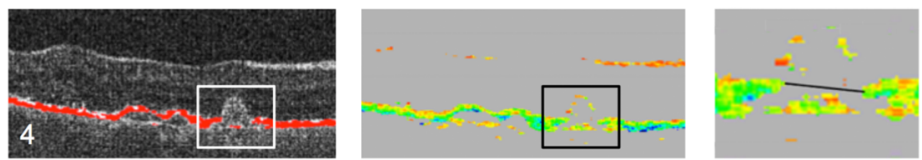

$0.32 \mathrm{~mm}$
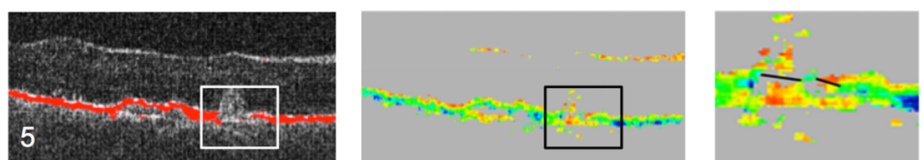

$0.25 \mathrm{~mm}$
Imaging procedures and properties of OCT devices

A PS-OCT prototype developed by the Center for Medical Physics and Biomedical Engineering, Medical University of Vienna, measuring multiple physical parameters simultaneously (reflectivity, retardation, optic axis orientation, degree of polarisation uniformity (DOPU)) $)^{12}$ was used for RPE and outer retinal layer imaging. ${ }^{13}$ The device includes a scanning laser ophthalmoscopy channel, acquiring fast online fundus images. High-resolution three-dimensional datasets are obtainable in 3.3 s. Raster scanning speed is $20000 \mathrm{~A}$-scans/s; axial resolution is $4.5 \mu \mathrm{m}$ in tissue. Three scanning patterns are available: $64 \times 1024, \quad 128 \times 512$ and $256 \times 256$ (B-scans $\times A$-scans). In this study, the $128 \times 512$ pattern was used, covering $6.2 \times 6.7 \times 3.3 \mathrm{~mm}^{3}$. The PS-OCT device operates at a centre wavelength of $839 \mathrm{~nm}$ with a full width at half maximum bandwidth of $58 \mathrm{~nm}$. Probe power is $700 \mu \mathrm{W}$ on the cornea. ${ }^{13}$

RPE segmentation is based on Stokes vector analysis. ${ }^{12}$ Stokes vector elements were derived from PS-OCT data, and averaged over adjacent pixels by calculating the mean value of each
Stokes vector element within a rectangular window. Then DOPU (a quantity related to the classical degree of polarisation) was calculated as a function of position. The RPE is characterised by low DOPU values, providing its selective differentiation. Based on a defined threshold of the DOPU parameter allowing for RPE segmentation in PS-OCT, areas of DOPU $<0.8$ were selected and used to generate an overlay of the segmented RPE onto the intensity image provided by PS-OCT. ${ }^{12}{ }^{13}$ This procedure enabled the visualisation of overlying retinal layers together with the RPE with a precise topography and demarcation.

In the DOPU images of figures $1-4$, depolarising material is represented in green to blue colour (ie, the RPE or depolarising material in outer retinal layers or at the RPE level), polarisation-preserving tissue (ie, the photoreceptor layer and inner retinal layers) is visualised in yellow, orange or red colour.

Cirrus intensity-based SD-OCT achieves $5 \mu \mathrm{m}$ axial resolution at $25000 \mathrm{~A}$-scans $/ \mathrm{s}$ (scanning area $=6 \times 6 \mathrm{~mm}^{2}$ ). The
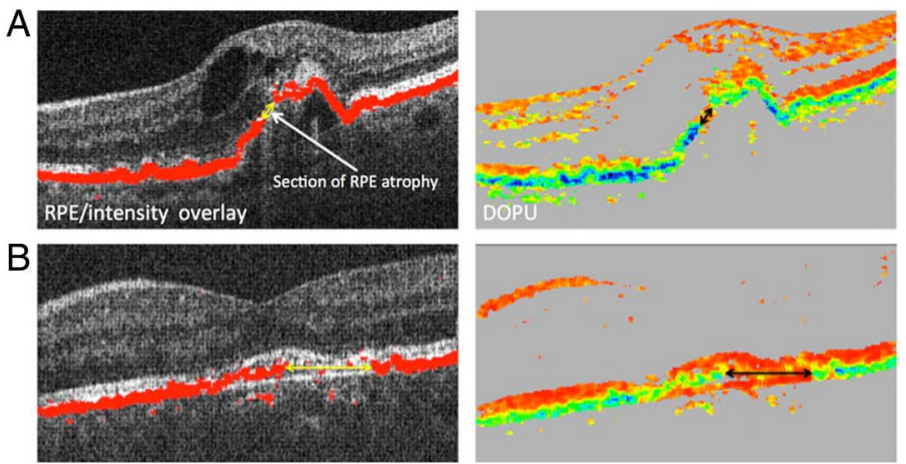
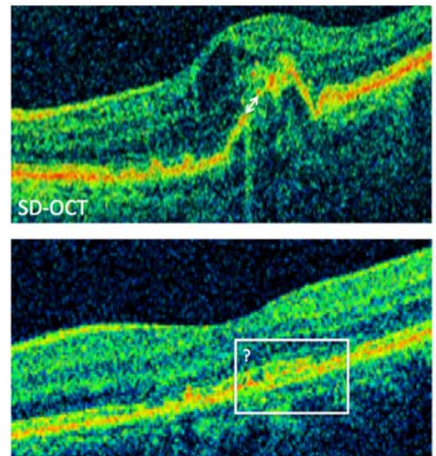

Figure 2 Comparison of RPE atrophy detection by PS-OCT and intensity-based SD-OCT in eyes with neovascular age-related macular degeneration. (A) shows the unambiguous identification of RPE atrophy in PS-OCT clearly corresponding to the intensity-based OCT images; (B) illustrates an example where the unambiguous identification of atrophic RPE is only possible using PS-OCT imaging. Note that in (B), it is not possible to clearly identify the borders of the atrophic zone (rectangle). RPE atrophy sections indicating RPE atrophy borders (arrows) are illustrated. DOPU, degree of polarisation uniformity; PS-OCT, polarisation-sensitive optical coherence tomography; RPE, retinal pigment epithelium; SD-OCT, spectral-domain optical coherence tomography. 
Figure 3 Qualitative reproducibility assessment of depolarising foci in outer retinal layers in a patient with neovascular age-related macular degeneration imaged by PS-OCT. All five repeated measurements (1-5) are shown. Note that regions of accumulations of depolarising material in outer retinal layers (circles) were repeatedly visualised by PS-OCT. shown (top left), revealing a depolarising material in PS-OCT. A retinal thickness map is illustrated at the top right. Depolarising hyper-reflective foci in outer retinal layers may represent RPE-derived material or melanin-containing polarisation uniformity; PS-OCT, polarisation-sensitive optical coherence tomography; RPE, retinal pigment epithelium; SD-OCT, spectral-domain optical coherence tomography. Further, an intensity SD-OCT image is correlation of hyper-reflective foci and macrophages. DOPU, degree of
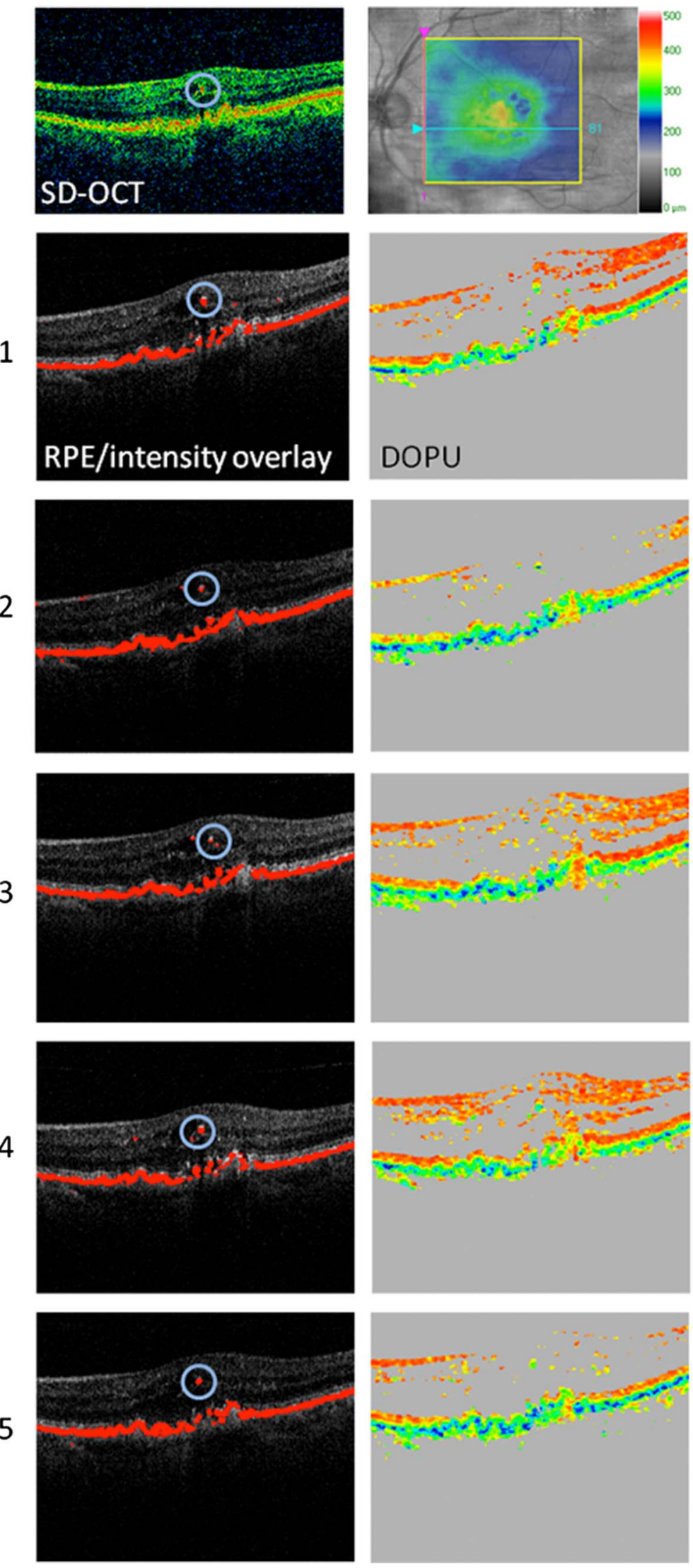

$512 \times 128 \times 1024$ scanning pattern was used. Cirrus SD-OCT was used in the context of the clinical evaluation of patients with neovascular AMD who were consequently included into this study.
Repeatability assessment of RPE atrophy section length using PS-OCT

To determine the repeatability of the section length of CNV-related RPE atrophy, five repeated measurements during a 
Figure 4 Qualitative reproducibility assessment of depolarising material accumulated at the level of the RPE in a patient with neovascular age-related macular degeneration imaged by PS-OCT. All five repeated measurements (1-5) are shown, representing identical B-scan locations. Regions of accumulations of depolarising material at the RPE level (blue circles) were imaged reproducibly by PS-OCT. The intensity-based SD-OCT image is shown, revealing increased hyper-reflectivity (orange circle), corresponding to accumulated depolarising material at the RPE level present in PS-OCT. However, a precise demarcation of RPE-related accumulations is only unambiguously identifiable by PS-OCT, not by intensity-based SD-OCT. DOPU, degree of polarisation uniformity; PS-OCT, polarisation-sensitive optical coherence tomography; RPE, retinal pigment epithelium; SD-OCT, spectral-domain optical coherence tomography.
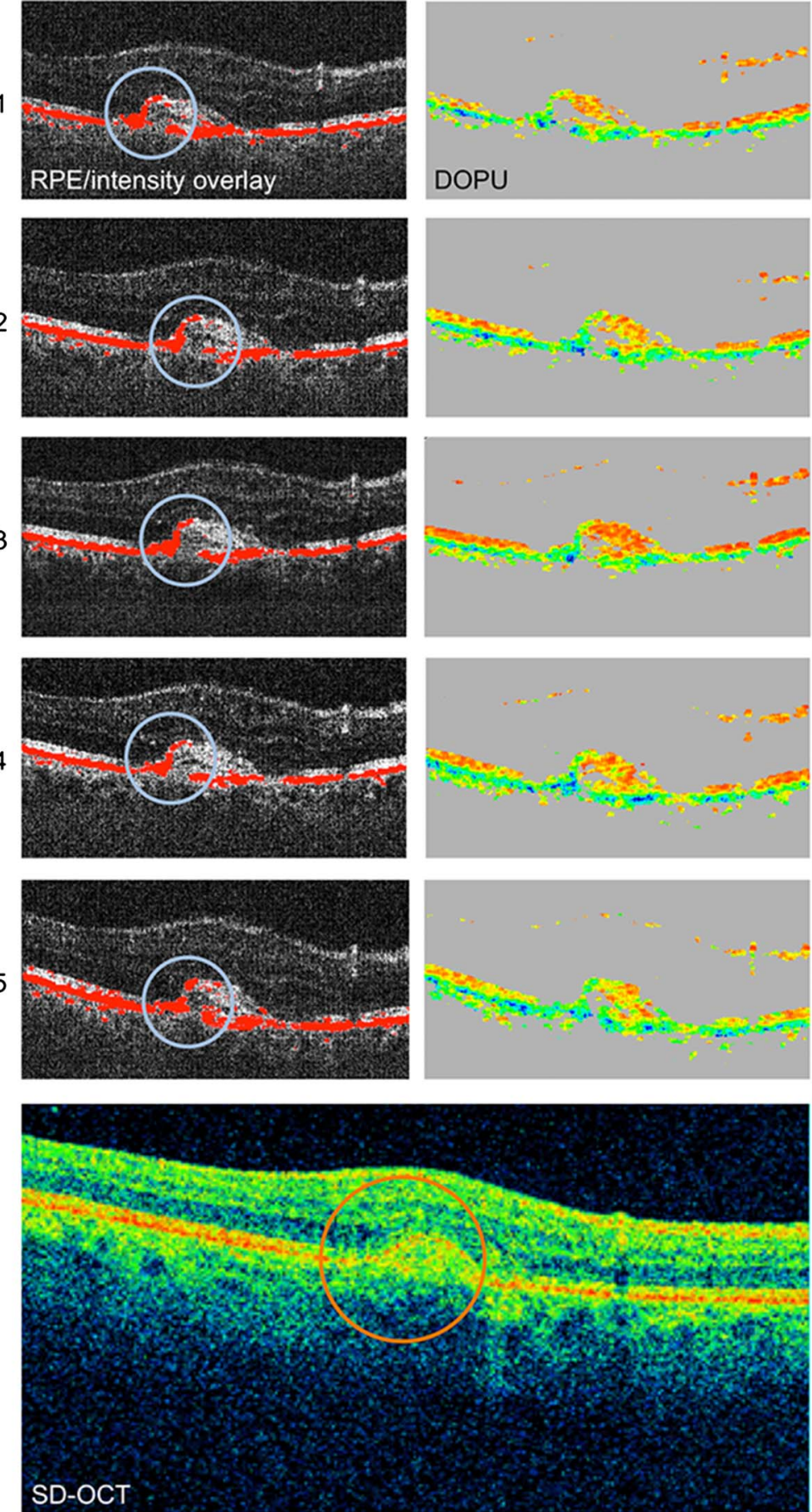

single visit were performed. RPE atrophy lesions with a minimum horizontal diameter of $100 \mu \mathrm{m}$ were included in our study. Following RPE segmentation, the respective B-scan located closest to the macular centre presenting with RPE atrophy was quantified manually using AutoCAD (US Metric). Thereby, the measurement of the linear diameter of RPE atrophy in five independent B-scans per examination and eye was performed. Reproducibility of RPE atrophy section lengths was assessed in each eye by comparing the size of RPE atrophy in a set of five individual measurements. The initial starting point of the analysed B-scan was determined at the first point of identifiable RPE atrophy starting from left to right. Subsequently, the cursor was set to the next spot where intact RPE was again observable. This procedure was followed for every atrophic zone in the B-scan until all RPE atrophy zones were included into the measurement process, sparing zones of intact RPE. By adding up all RPE atrophy distances per B-scan, a final result per measurement was obtained. Two independent readers evaluated interobserver agreement.

Qualitative reproducibility of accumulating depolarising material present (accumulations of depolarising material at the RPE level and in outer retinal layers) was assessed by analysing five repeated PS-OCT datasets per eye in all eyes included into this study. Intensity-based SD-OCT images revealing hyper-reflective foci in outer retinal layers and hyper-reflective lesions at the RPE level were compared with PS-OCT images concerning correlations of depolarising material at 
corresponding locations. Both readers performed the qualitative analysis.

DOPU images were primarily used for identifying regions of RPE atrophy and accumulations of depolarising material, as DOPU images represent the condition of the RPE and related alterations most accurately, and are the basis for generating overlays of the segmented RPE onto the intensity image. Overlays of the segmented RPE and related depolarising structures with the intensity scan are also presented in the current study to make results most apprehensive to the reader.

PS-OCT images were compared with corresponding Cirrus OCT images regarding RPE atrophy identification.

\section{Data analysis}

Manual RPE atrophy size evaluation was performed in a total of 140 PS-OCT B-scans. For reproducibility analyses, mean values, $\mathrm{SD}(\mathrm{SD} \pm$ ) and a coefficient of variation analysis ( $\mathrm{SD} \pm /$ mean) were used. Mean variability of all RPE atrophy section lengths was calculated. The variability describes the value of the minimum atrophy size subtracted from the maximum value measured within the five scans recorded per eye. Interobserver agreement was evaluated applying the paired t test and the Spearman correlation coefficient. Regarding the comparison analysis of RPE lesions identified by PS-OCT with conventional intensity-based Cirrus-OCT imaging, the percentage of eyes where zones of RPE atrophy corresponded clearly to PS-OCT images was assessed. Statistical significance was defined with $\mathrm{p}<0.05$. Statistical software used was Med Calc V.11.5.1.0 and Excel 2011.

\section{RESULTS}

Mean age of patients was 81 years (SD $\pm=7.6$ years); 18 patients were female; 8 were male. Thirteen right eyes and 15 left eyes were included. Mean BCVA was 0.46 Snellen (range 0.05-0.8). Two patients saw hand movements; two patients were able to count fingers.

Mean RPE atrophy dimensions of all eyes (of all 140 B-scans) detected by PS-OCT were $0.74 \mathrm{~mm} \quad(S D \pm=0.55 \mathrm{~mm}$, median $=0.66 \mathrm{~mm}$, range $0.14-2.06 \mathrm{~mm})$. Mean variability of all atrophy section lengths was $0.10 \mathrm{~mm} \quad(S D \pm=0.06 \mathrm{~mm})$. Average coefficient of variation ( $\mathrm{SD} \pm /$ mean) was 0.06 $(\mathrm{SD} \pm=0.03)$. No outliers or far-out values were statistically identifiable. Results are summarised in table 1.

Figure 1 shows a representative example of a 73-year-old female patient with neovascular AMD and respective PS-OCT images revealing RPE atrophy. All five measurements, including manually quantified RPE atrophy dimensions are shown. The DOPU images offer specific RPE identification due to its intrinsic depolarising properties. The RPE is shown as a greenish band. In the atrophic zone, the green band is missing. The respective atrophy section length was repeatedly manually quantified regarding its linear diameter (black line). Mean atrophy section length was $0.31 \mathrm{~mm}(\mathrm{SD} \pm=0.04 \mathrm{~mm})$ in this patient, the coefficient of variation was 0.13 and variability of atrophy section lengths was $0.11 \mathrm{~mm}$, representing good reproducibility of $\sim 90 \%$.

Mean RPE atrophy size in untreated eyes was $0.78 \mathrm{~mm}$ (SD $\pm=0.57 \mathrm{~mm})$ and $0.52 \mathrm{~mm}(\mathrm{SD} \pm=0.34 \mathrm{~mm})$ in treated eyes. Mean variability of RPE atrophy section lengths in the untreated group was $0.10 \mathrm{~mm} \quad(\mathrm{SD} \pm=0.06 \mathrm{~mm})$ and $0.06 \mathrm{~mm}$ (SD $\pm=0.03 \mathrm{~mm})$ in the treated group. Coefficients of variation in the untreated and treated groups were $0.06(\mathrm{SD} \pm=0.03)$ and $0.05(\mathrm{SD} \pm=0.02)$, respectively.
Table 1 RPE atrophy section lengths detected by PS-OCT in 26 patients (28 eyes) with CNV related to neovascular AMD

\begin{tabular}{|c|c|c|c|}
\hline Eye no/eyes & $\begin{array}{l}\text { Mean lesion section } \\
\text { length }(\mathrm{mm})\end{array}$ & $\mathrm{SD}(\mathrm{mm})$ & $\begin{array}{l}\text { Coefficient } \\
\text { of variation }\end{array}$ \\
\hline 1 & 0.14 & 0.01 & 0.05 \\
\hline 2 & 0.24 & 0.02 & 0.07 \\
\hline 3 & 1.31 & 0.05 & 0.04 \\
\hline 4 & 0.47 & 0.03 & 0.06 \\
\hline 5 & 0.31 & 0.04 & 0.13 \\
\hline 6 & 0.51 & 0.04 & 0.08 \\
\hline 7 & 0.20 & 0.02 & 0.11 \\
\hline 8 & 0.97 & 0.02 & 0.02 \\
\hline 9 & 1.72 & 0.06 & 0.03 \\
\hline 10 & 1.92 & 0.07 & 0.03 \\
\hline 11 & 1.40 & 0.04 & 0.03 \\
\hline 12 & 0.62 & 0.05 & 0.09 \\
\hline 13 & 0.30 & 0.03 & 0.11 \\
\hline 14 & 0.52 & 0.03 & 0.06 \\
\hline 15 & 0.63 & 0.03 & 0.05 \\
\hline 16 & 1.60 & 0.06 & 0.03 \\
\hline 17 & 0.64 & 0.05 & 0.08 \\
\hline 18 & 0.37 & 0.03 & 0.09 \\
\hline 19 & 0.18 & 0.01 & 0.04 \\
\hline 20 & 0.65 & 0.04 & 0.05 \\
\hline 21 & 0.29 & 0.02 & 0.06 \\
\hline 22 & 0.96 & 0.04 & 0.04 \\
\hline 23 & 0.31 & 0.01 & 0.03 \\
\hline 24 & 0.43 & 0.02 & 0.04 \\
\hline 25 & 0.74 & 0.10 & 0.14 \\
\hline 26 & 2.06 & 0.09 & 0.05 \\
\hline 27 & 0.58 & 0.07 & 0.12 \\
\hline 28 & 0.67 & 0.03 & 0.04 \\
\hline Average & 0.74 & 0.04 & 0.06 \\
\hline Min & 0.14 & 0.01 & 0.02 \\
\hline Max & 2.06 & 0.10 & 0.14 \\
\hline
\end{tabular}

AMD, age-related macular degeneration; CNV, choroidal neovascularisation; PS-OCT, polarisation-sensitive optical coherence tomography; RPE, retinal pigment epithelium.

PS-OCT unambiguously identified the RPE and associated atrophy in all eyes compared with conventional intensity-based SD-OCT that did not clearly identify borders of the atrophic zone in 25 eyes $(89.3 \%, \mathrm{p}<0.01$, figure 2$)$.

\section{Interobserver variability}

Differences of RPE atrophy measurements of all eyes were not statistically significant between both readers $(p=0.38)$. Spearman correlation coefficient was $\mathrm{r}=0.98$ with $\mathrm{p}<0.001$ (slope of the trend line $=0.98$ ). Mean RPE atrophy size of all eyes was $0.74 \mathrm{~mm}(\mathrm{SD} \pm=0.55$, range $0.14-2.06$, coefficient of variation $=0.06(\mathrm{SD} \pm=0.03))$ for reader 1 and $0.76 \mathrm{~mm}$ (SD $\pm=0.55$, range $0.14-2.09$, coefficient of variation $=0.08$ $(\mathrm{SD} \pm=0.05))$ for reader 2 , respectively.

In addition to RPE atrophy, our patients frequently revealed accumulations of depolarising material in outer retinal layers, potentially related to intraretinal macrophages and exudates (particularly at the level of the outer nuclear layer) (figure 3). Accumulations of depolarising material were further found at the RPE level, possibly depicting the latter's potential of proliferative activity (figure 4). Accumulations of depolarising material were imaged reproducibly in all PS-OCT datasets, and correlated well with hyper-reflective foci in SD-OCT (figure 3). 


\section{DISCUSSION}

Comparability, repeatability and objectivity are essential factors when implementing a new method and novel diagnostic approaches.

Previous investigations showed that PS-OCT provides precise identification of the RPE in AMD. ${ }^{11} 131417$ However, data confirming the repeatability of the method in neovascular AMD are still missing (essential to assess its clinical applicability). The present study evaluates the reproducibility of RPE atrophy quantification in patients with neovascular AMD using PS-OCT. Further, the qualitative reproducibility of the detection of depolarising material at the RPE level and within retinal structures by PS-OCT is analysed.

Our results showed an average repeatability of RPE atrophy size measurements of $\sim 90 \%$, signifying good reproducibility. Moreover, interobserver agreement was excellent (Spearman correlation coefficient $\mathrm{r}=0.98$ with $\mathrm{p}<0.001$ ), confirming the objectivity of PS-OCT when applied for RPE atrophy detection in CNV.

Further, the similar reproducibility of RPE atrophy section lengths in the untreated and the treated group indicates that RPE atrophy measurements in PS-OCT are reproducible in patients with different disease stages, independent of treatment intervention. (Although, patient numbers in the untreated group were too small to reliably determine statistical significance between the treated and untreated group).

This study also showed that PS-OCT reproducibly detects accumulations of depolarising material in outer retinal layers (corresponding to hyper-reflective foci in intensity-based SD-OCT, figure 3) and at the RPE level (figure 4), possibly representing migrating RPE cells and exudates. These results are relevant as previous in vitro histological studies have showed that RPE recovery is accomplished by RPE proliferation and hyperplasia. ${ }^{18}$ The reproducible in vivo detection of these RPE-related alterations, therefore, is of high scientific and clinical value. These findings make the method potentially suitable for the analysis of pathologically affected RPE regarding the latter's loss and/or accumulation.

Although it has been shown previously that conventional SD-OCT imaging is applicable for evaluating GA in dry AMD,${ }^{19-22}$ less work has been done describing RPE atrophy quantitatively in neovascular AMD. ${ }^{23}$ The assessment of atrophy dimensions by RPE-specific PS-OCT imaging, as performed in the current study, allowed to demonstrate the advantages of PS-OCT, evaluating RPE lesions related to neovascular AMD reproducibly, compared with standard intensity-based SD-OCT monitoring, showing limitations in clearly detecting the borders of the atrophic zone in the majority of eyes (figure 2). Apart from the RPE specificity of PS-OCT, another reason for this may be that RPE atrophy in CNV is partially very small, different to GA in dry AMD (characterised by circumscribed RPE atrophy of at least $250 \mu \mathrm{m}$ in longest diameter), ${ }^{24}$ making the differentiation of atrophic RPE in intensity-based SD-OCT particularly difficult due to layers with similar reflectivity.

This study may further be used as a cornerstone for the evaluation of RPE atrophy progression during follow-up in patients with CNV treated with anti-vascular endothelial growth factor (anti-VEGF), enhancing our understanding about disease-specific pathophysiological characteristics. Detection of subtle RPE atrophy (ie, in the beginning of the treatment phase with anti-VEGF in patients with neovascular AMD) or other lesions (ie, accumulations of depolarising material at the RPE level and in outer retinal layers) may provide considerable impact in the estimation of prognosis regarding the prospective condition of the RPE of an individual patient. Nevertheless, this remains to be investigated in larger prospective clinical trials.

Our group ${ }^{13}$ has recently shown that PS-OCT achieves high reproducibility of automatic RPE atrophy size determination in GA (coefficient of variation was 0.07 on average (range $0.01-$ 0.19 ) revealing a repeatability of $\sim 90 \%)$. The findings of the current study and of previous investigations, therefore, underline the potential of the clinical applicability of PS-OCT in neovascular and dry AMD.

A limitation of the present study is that RPE atrophy size was obtained manually, instead of using automatic atrophyquantification algorithms. The integration of an eye tracker function would potentially improve repeatability of measurements in next-generation PS-OCT devices. ${ }^{5}$ Although only one central PS-OCT B-scan has been used for reproducibility analysis, this method seems justified as similar approaches have been used in previous studies. ${ }^{25}$ Automated en-face assessment of RPE atrophy area in CNV, similar as recently shown in patients with GA in dry AMD,${ }^{13}$ would be desirable, as this approach would allow for a precise topographic analysis of the full atrophy dimensions imaged in an entire PS-OCT dataset. Further, patterns of the partially diffusely distributed RPE atrophies could be analysed using the RPE specificity of PS-OCT, improving our understanding about RPE atrophy characteristics and the RPE-related disease course of CNV. Automated PS-OCT segmentation algorithms are, however, presently not designed to assess RPE atrophy dimensions planimetrically in CNV yet, as recently shown for GA. ${ }^{13}{ }^{17}$ Algorithms automatically measuring the whole area of RPE atrophy in CNV are currently being developed, and will be used in future investigations.

In conclusion, PS-OCT proved evidence of good reproducibility in RPE atrophy quantification related to neovascular AMD with an excellent interobserver agreement. Further studies are needed to analyse the reproducibility of RPE atrophy dimensions in different retinal diseases involving RPE atrophy (ie, macular dystrophies or myopia) to demonstrate the full clinical potential of PS-OCT.

Acknowledgements The critical analysis and editing of this manuscript by Aner Gurvitz is gratefully acknowledged.

Contributors The study was conducted by CS, KT, BB, MP, CKH and US-E; data collection and data management were performed by $\mathrm{CS}$ and $\mathrm{KT}$; data was analysed by $\mathrm{CS}$; interpretation of the data was done by $\mathrm{CS}, \mathrm{KT}, \mathrm{BB}, \mathrm{MP}, \mathrm{CKH}$, EG and US-E; the manuscript was prepared and written by $C S$; critical revision of the manuscript was performed by CKH and US-E.

Funding This study was supported by an independent scientific grant (FWF grant P19624-B02, Austrian Science Fund) and the European Union (FP7 HEALTH programme grant 201880, FUN-OCT).

Competing interests None declared.

Patient consent Obtained.

Ethics approval Ethics Committee, Medical University of Vienna.

Provenance and peer review Not commissioned; externally peer reviewed.

Open Access This is an Open Access article distributed in accordance with the Creative Commons Attribution Non Commercial (CC BY-NC 4.0) license, which permits others to distribute, remix, adapt, build upon this work non-commercially, and license their derivative works on different terms, provided the original work is properly cited and the use is non-commercial. See: http://creativecommons.org/ licenses/by-nc/4.0/

\section{REFERENCES}

1 Yin $\mathrm{L}, \mathrm{Wu} X$, Gong $Y$, et al. OX-LDL up regulates the vascular endothelial growth factor-to pigment epithelium-derived factor ratio in human retinal pigment epithelial cells. Curr Eye Res 2011;36:379-85. 
2 Biasutto L, Chiechi A, Couch R, et al. Retinal pigment epithelium (RPE) exosomes contain signaling phosphoproteins affected by oxidative stress. Exp Cell Res 2013;319:2113-23.

3 Ding $X$, Patel M, Chan CC. Molecular pathology of age-related macular degeneration. Prog Retin Eye Res 2009;28:1-18.

4 Braaf B, Vermeer KA, de Groot M, et al. Fiber-based polarization-sensitive OCT of the human retina with correction of system polarization distortions. Biomed Opt Express 2014;5:2736-58.

5 Sugita M, Zotter S, Pircher M, et al. Motion artifact and speckle noise reduction in polarization sensitive optical coherence tomography by retinal tracking. Biomed Opt Express 2013;5:106-22.

6 Yamanari M, Lim Y, Makita S, et al. Visualization of phase retardation of deep posterior eye by polarization-sensitive swept-source optical coherence tomography with 1-micron probe. Opt Express 2009;17:12385-96.

7 Cense B, Gao W, Brown JM, et al. Retinal imaging with polarization-sensitive optical coherence tomography and adaptive optics. Opt Express 2009;17: 21634-51.

8 Pircher M, Hitzenberger CK, Schmidt-Erfurth U. Polarization sensitive optical coherence tomography in the human eye. Prog Retin Eye Res 2011;30:431-51.

9 Götzinger E, Pircher M, Hitzenberger CK. High speed spectral domain polarization sensitive optical coherence tomography of the human retina. Opt Express 2005; 13:10217-29.

10 Pircher M, Götzinger E, Findl O, et al. Human macula investigated in vivo with polarization-sensitive optical coherence tomography. Invest Ophthalmol Vis Sci 2006;47:5487-94.

11 Ahlers $C$, Götzinger $E$, Pircher $M$, et al. Imaging of the retinal pigment epithelium in age-related macular degeneration using polarization-sensitive optical coherence tomography. Invest Ophthalmol Vis Sci 2010;51:2149-57.

12 Götzinger E, Pircher M, Geitzenauer W, et al. Retinal pigment epithelium segmentation by polarization sensitive optical coherence tomography. Opt Express 2008; 16:16410-22

13 Baumann B, Gotzinger $E$, Pircher $M$, et al. Segmentation and quantification of retinal lesions in age-related macular degeneration using polarization-sensitive optical coherence tomography. J Biomed Opt 2010;15:061704.
14 Schlanitz FG, Baumann B, Spalek T, et al. Performance of automated drusen detection by polarization-sensitive optical coherence tomography. Invest Ophthalmol Vis Sci 2011;52:4571-9.

15 Pircher M, Goetzinger $\mathrm{E}$, Leitgeb R, et al. Transversal phase resolved polarization sensitive optical coherence tomography. Phys Med Biol 2004;49:1257-63.

16 Götzinger E, Pircher M, Baumann B, et al. Three-dimensional polarization sensitive OCT imaging and interactive display of the human retina. Opt Express 2009:17:4151-65.

17 Schütze C, Bolz M, Sayegh R, et al. Lesion size detection in geographic atrophy by polarization sensitive optical coherence tomography and correlation to conventional imaging techniques. Invest Ophthalmol Vis Sci 2013;54:739-45.

18 Tsuboi S, Pederson JE, Toris CB. Functional recovery of retinal pigment epithelial damage in experimental retinal detachment. Invest Ophthalmol Vis Sci 1987;28:1788-94.

19 Simader C, Sayegh RG, Montuoro A, et al. A longitudinal comparison of spectral-domain optical coherence tomography and fundus autofluorescence in geographic atrophy. Am J Ophthalmol 2014;158:557-66.

20 Chen Q, de Sisternes L, Leng T, et al. Semi-automatic geographic atrophy segmentation for SD-OCT images. Biomed Opt Express 2013;4:2729-50.

$21 \mathrm{Hu}$ Z, Medioni GG, Hernandez M, et al. Segmentation of the geographic atrophy in spectral-domain optical coherence tomography and fundus autofluorescence images. Invest Ophthalmol Vis Sci 2013;54:8375-83.

22 Yehoshua Z, Garcia Filho CA, Penha FM, et al. Comparison of geographic atrophy measurements from the OCT fundus image and the sub-RPE slab image. Ophthalmic Surg Lasers Imaging Retina 2013;44:127-32.

23 Channa R, Sophie R, Bagheri S, et al. Regression of choroidal neovascularization results in macular atrophy in anti-vascular endothelial growth factor-treated eyes. Am J Ophthalmol 2014;159:9-19.

24 Grunwald JE, Daniel E, Huang J, et al, CATT Research Group. Risk of geographic atrophy in the comparison of age-related macular degeneration treatments trials. Ophthalmology 2014;121:150-61.

25 Rahman W, Chen FK, Yeoh J, et al. Repeatability of manual subfoveal choroidal thickness measurements in healthy subjects using the technique of enhanced depth imaging optical coherence tomography. Invest Ophthalmol Vis Sci 2011;52:2267-71. 